\title{
SOURCE LOCALIZATION IN A WEDGE SHAPED SHALLOW SEA
}

\author{
P.S. Naidu and H.R. Udaya Shankar, \\ Department of Electrical Communication Engineering, \\ Indian Institute of Science, \\ Bangalore 560012
}

\begin{abstract}
This paper looks into the problem of localization of an acoustic source in shallow sea with a sloping bottom as found in the continental shelf area. A vertical array of sensors, either floating or secured to the sea bottom, is used to receive the acoustic signals from the source including those travelling by different paths. First, a spectral matrix of the array signal is computed and it is then subjected to eigenvalue decomposition. The space spanned by the eigenvectors is partitioned into two subspaces, namely, a signal subspace spanned by the eigenvector corresponding to largest eigenvalues and a noise subspace spanned by the eigenvectors corresponding to repeated eigenvalues. The signal subspace is also spanned by a linear combination of direction vectors corresponding to all multipaths These two subspaces are orthogonal. We exploit this property of orthogonality in the estimation of range, bearing and depth of a source. Some of the significant findings are as follows:(1) Unlike in the flat bottom channel it is now possible to measure the bearing with respect to upslope or downslope direction.(2) The azimuth scan shows a sharp peak at the correct location for steep slope $\left(>1^{\circ}\right.$ ) (3) A study of finite data effect has shown that the background generally remains unchanged as the number of snapshots increases. However, the signal peak amplitude increases roughly at the rate of $3 \mathrm{~dB}$ per doubling of the number of snapshots.
\end{abstract}

\section{Introduction:}

In under water applications it is of considerable interest to locate a sound source, such as, an underwater vehicle. The problem of localization becomes significantly difficult, when the source is in the shallow water region, since the energy can now reach the receiver array via several paths, causing considerable confusion when a simple minded delay-and-sum beamformer is used. The location coordinates, viz., range, azimuth and depth, are embedded in the expression of sound field in a nonlinear form, making their estimation from the observed data difficult.

Recently Clay[1] suggested a time domain technique for the source localization in shallow water, using a parallel waveguide as a model. His method makes use of channel impulse response

between the source and the receiver for inverse filtering. He developed a match filter for each sensor output whose impulse response is defined by the inverse of the channel impulse function at the sensor for a given source position. The cross-correlation of the outputs of the matched filters are taken pairwise, and averaged, over a grid to obtain global maximum, for the source location estimation. He reported that the ambiguity of the source location decreases with the increase in the complexity of impulse response and that the spectral resolution and the signal to noise gain depeii. $s$ on the time bandwidth product of the data record.These authors[2] have used this method to localize a source in a wedge shaped waveguide. Laboratory measurements were made in the air wedge having rigid sheet rock boundaries.. The wedge angle in the experiment was $6.6^{\circ}$. Source transmited a short oscillatory signal having a frequency band of 3 $13 \mathrm{kHz}$. The performance of the source location technique depended on the accuracy of the numerical calculation of the source-to-receiver transmission response and the bandwidth of the source.

Multi image subspace algorithm(MISA) was developed by Mohan[3] for a Pekeris channel, that is. sea water layer resting on horizontal sedimentary layer which is considered as a fluid. But, in the continental margin the sea bottom may be approximated by two or more sloping surfaces as shown in Fig. 1. This causes a mismatch between the model used and the actual situation. It is observed that the MISA is very sensitive to such mismatch (see Fig.2). In this paper we propose to model the shallow sea in the continental margin region as a wedge shaped water layer resting on soft sediments. The sea bottom consists of steeply dipping $15^{\circ}$ to $\left.10^{\circ}\right)$ continental margin and flat bottom deep sea.

\section{Theoretical Analysis:}

\section{a) MISA for flat bottom channel:}

Consider a uniform channel of depth $\mathrm{H}$ wherein a vertical array of equispaced (sensor spacing $<\lambda / 2$ ) sensors located at a depth $Z_{R}$ (depth is measured from the top of the channel to the top sensor of the array Fig.3). Let there be a narrow band sound source $S_{01}$ at a depth $Z_{S}$ and horizontally separated by a distance $R_{0}$ from the array. Water surface is perfectly compliant, with the reflection coefficient $\left(V_{S}=-1\right)$. The sound source $S_{01}$ and its reflection $\mathrm{SO}_{2}$ can be considered as a vertical dipole source. The presence of the sea bottom produces its image dipole ( $\mathrm{S}_{03}-\mathrm{S}_{04}$ ) below the bottom interface. This is in turn reflected by the surface producing another dipole image above the surface. This process is repeated producing an infinite number of images along the vertical line passing through the source Sol. The vertical separation from the receiver to the image $S_{i j}$ for $i=0,1 \ldots \ldots$ and $j=1,2,3,4$ is given by

$$
\begin{aligned}
& Z_{i 1}=2 i H+Z_{R}-Z_{s} \\
& Z_{i 2}=2 i H+Z_{R}+Z_{s} \\
& Z_{i 3}=2(i+1) H-Z_{R}-Z_{s} \\
& Z_{i 4}=2(i+1) H+Z_{R}-Z_{s}
\end{aligned}
$$

The slant distance from $S_{i j}$ to the receiver is 
$R_{i 1}=\sqrt{R_{0}^{2}+Z_{i, 1}^{2}}$. The arrival angle of the image $S_{i j}$ measured with respect to horizontal is $\theta_{i 11}=\tan ^{-1}\left(Z_{i j} / R_{0}\right)$. Since we have considered a soft bottom channel for the model, the reflection coefficient $\mathrm{V}_{b}$ is given by

$$
V_{b}\left(\theta_{i j}\right)=\frac{m \sin \left(\theta_{i j}\right)-\sqrt{n^{2}-\cos ^{2}\left(\theta_{i j}\right)}}{m \sin \left(\theta_{i j}\right)+\sqrt{n^{2}-\cos ^{2}\left(\theta_{i j}\right)}}
$$

where $m=\frac{p_{1}}{p_{0}}, \quad n=\frac{c_{1}}{c_{0}}, \quad r 1=$ density of sea water, $r b=$ density of sediments, $c_{1}=$ acoustic velocity in sea water and $\mathrm{Cb}$ acoustic velocity in sediments.

The strength of the image $S_{i j}$ at the receiver, for normalized source power denoted by $\alpha_{11}$, is a function of the number of reflections that the ray from Sol undergoes at each boundary with reflection coefficient $V_{S}$ and $V_{b}$, at the surface and at the bottom respectively. They are given by

$$
\begin{aligned}
& \alpha_{11}=\left(v_{s} v_{b}\right)^{\prime} \\
& \alpha_{12}=\left(V_{s} v_{b}\right)^{\prime} \cdot v_{s} \\
& \alpha_{13}=\left(V_{s} v_{b}\right)^{\prime} \cdot v_{b} \\
& \alpha_{14}=\left(V_{s} v_{b}\right)^{i+1}
\end{aligned}
$$

The wavefronts arriving at angle greater than the critical angle under go multiple reflections at the bottom with the reflection coefficient whose magnitude is less than unity. Hence strength of the dipole decreases as arrival angle increases. The contribution from the distant images is further reduced because of cylindrical spreading and the medium attenuation, if there is any. Consequently, only a small number of images out of infinite set, will contribute to the total acoustic power received by the array. For practical purposes, significant images are those images whose strength is greater than atleast $1 \%$ of the strength of the primary source. For any channel it is possible to estimate this number for given range. In the sequel this number will be denoted by $M$.

The waveform received from these $M$ images at $1^{\text {th }}$ sensor ( $1=0$ is the top sensor) is given by

$$
\begin{gathered}
X_{1}(t)=\sum_{i=1}^{m} \frac{\alpha_{1}}{\sqrt{R_{i}}} x_{0}\left(t-\tau_{i i}\right)+\eta_{1}(t) \\
l=0.1, \ldots N-1
\end{gathered}
$$

where $X_{0}(t)$ is the signal emitted by the souree, presumed to be a stationary stochastic process, $\tau_{\| 1}$ is the delay from ith image, $R_{1}$ is distance to the $1^{\text {th }}$ image and $\alpha_{1}$ the amplitude of the signal arriving from the ith image. $\eta_{1}(t)$ is the noise received (assumed to be white) by the $1^{\text {th }}$ sensor. It is easy to show that.

$$
\tau_{11}=\tau_{1}+\frac{d_{0}}{c} l \sin \left(\theta_{1}\right) \quad \mid=0,1, \ldots N-1
$$

where $t_{i}$ is travel time from $i^{\text {th }}$ image to the top sensor and $\theta_{1}$ is angle (with respect to the horizontal) of the wave front from ith image and $c$ is sound velocity in sea water, using the spectral representation of a stationary stochastic process the above equation may be written as

$d X_{1}(\omega)=\sum_{i=1}^{M} W_{1} d X_{0}(\omega) \exp \left(j \frac{2 \pi d}{\lambda}(1-1) \sin \left(\theta_{1}\right)\right)+d \eta_{1}(\omega)$

where $W_{1}=\frac{\alpha_{1}}{R_{1}} e^{-j \omega t}$. We have used the spectral representation of stationary stochastic process[4] in the above derivation.

$$
X_{0}(t)=\int_{-\pi}^{\pi} d X_{0}(\omega) \exp (j \omega t)
$$

Define the following vectors and matrices:

$$
\begin{aligned}
& d X(\omega)=\left[\begin{array}{llll}
d X_{0}(\omega) \cdot d X_{1}(\omega) & \ldots & d X_{n}(\omega)
\end{array}\right]^{\top} \\
& \mathbf{w}=\left[\mathbf{W}_{1}, \mathrm{~W}_{2}, \ldots \ldots \ldots \ldots \ldots \mathrm{W}_{\mathrm{M}}\right]^{\mathrm{T}}
\end{aligned}
$$

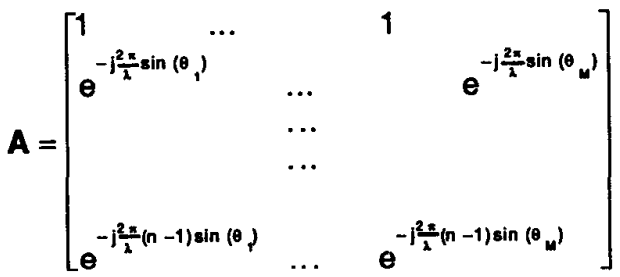

and

$$
d \eta(\omega)=\left[\begin{array}{llll}
d \eta_{0}(\omega), d \eta_{1}(\omega) & \ldots & d \eta_{n-1}(w)
\end{array}\right]^{\top}
$$

In the matrix form $\mathrm{Eq}(2)$ becomes

$$
d X(w)=A W d x_{0}(w)+d \eta(w)
$$

The spectral matrix is obtained from Eq(3)

$$
S_{x}(\omega)=A W W^{H} A^{H} S_{0}(\omega)+\sigma_{n}^{2} I
$$

where Solw) (scalar) is spectrum of the source and $\sigma_{n}^{2}$ is the variance of the background noise assumed to be white and uncorrelated.

The the column space of the matrix $\mathbf{s}_{\mathbf{x}}(\mathbf{w})$ can be split into two orthogonal subspaces viz., signal subspace and the noise subspace. It can be shown that noise subspace is orhogonal to the vector $\mathbf{A W}$, at the correct source location. We exploit this property for the source parameter estimation purpose. We compute the following equitation over complete parameter space,

$$
S(r, \not)=\frac{1}{\left[W_{1}^{H} A_{1}^{H} E_{N} E_{N}^{H} A_{1} W_{1}\right]}
$$


$A_{1} W_{1}$ is steering vector formed over the complete parameter space $[r, z]$. $S(r, z)$ has an infinite value at the true $(r, z)$, in the asymptotic case.

\section{b. MISA for Sloping bottom}

It was shown in the previous section that an acoustic source in a shallow water channel would produce several images. When the sea bottom is flat (which is most unlikely), the sea surface and bottom makes a pair of parallel mirrors. In this arrangement a source between the two mirrors would produce varietally infinite number of images, all of which lie on a vertical line passing though the source. But the situation drastically changes when the sea bottom is sloping (which is most likely). Now the reflecting surface and the bottom would make a pair of angled mirrors and in this arrangement the source between them would produce only a finite number of images which would lie on a circle passing through the source. The center of the circle is at the apex of the wedge (meeting point of two mirrors).

The image distribution in a sloping bottom channel for slope of $b=45^{\circ}$ and when the source is downslope is shown in Fig. 4. The ray emitted by $\mathrm{S}_{21}$, which is an image of $\mathrm{S} 13$ in the surface, does not reach the bottom, hence this will not produce any further images. Similarly, a ray emitted by the image $S_{14}$, which is an image of $\mathrm{S}_{12}$ in the bottom. does not reach the surface and hence it would not form any further images. Thus, the number of images (including the source) is equal to $360 / \beta$ when $\beta$ is an integer sub-multiple of $180^{\circ}$ and it is equal to $(360 / \beta+1)$ when $\beta$ is a noninteger submultiple. This is because, when $\beta$ is integer submultiple of 180 , the images $S_{21}, S_{14}$ would overlap. It should be observed that depending on the receiver position either $S_{14}$ or $S_{21}$ or both can be seen. This is depicted in the Fig. 4, which shows the raytraces of the image $\left(S_{21}, S_{14}\right)$ for two receiver positions. The receiver \#1 can see the image $S_{21}$ which is a reflection of $\mathrm{S}_{13}$ in the surface, but not the image S14, which is a reflection of $\mathrm{S}_{12}$ in the bottom. The receiver \#2 can see the image $S_{14}$ but not $S_{21}$.

It can be observed from Fig 5 that grazing angle increases after each bounce at the bottom, when the source is down slope direction. Then the reflection coefficients are given by,

$$
\begin{aligned}
& \alpha_{i 1}=\left(V_{s}\right)^{i} \prod_{k=1}^{i}\left(\theta_{i j}+(2 k-1) \beta\right) \\
& \alpha_{i 2}=\left(V_{s}\right)^{i+1} \prod_{k=1}^{i}\left(\theta_{i j}+(2 k-1) \beta\right) \\
& \alpha_{i 3}=\left(V_{s}\right) \prod_{k=1}^{i+1}\left(\theta_{i j}+(2 k-1) \beta\right) \\
& \alpha_{i 4}=\left(V_{s}\right)^{i+1} \prod_{k=1}^{i+1}\left(\theta_{i j}+(2 k-1) \beta\right)
\end{aligned}
$$

where $\beta$ is the wedge angle.If the source is in the upslope direction as in fig $5 \mathrm{~b}$, the grazing angle decreases after each bounce.
It is of interest to note that, when the source is in down slope direction as shown in Fig. 4, the receiver is surround by a circle of images and the ray from the images which lies to the left of the receiver travel from right to left crossing the receiver position and then returns back to the source after several reflections from the top and the bottom. When the source is in upslope all the images lie on the left side of the receiver and none of the rays travel ahead of the receiver.

In the case of a flat bottom channel, all the images are on a vertical line and hence there is radial symmetry. Since a vertical array which is made of ommi directional equispaced sensors, is insensitive to the azimuth angle, it is not possible to extract the azimuth information from a single vertical array. This radial symmetry is lost in the case of sloping bottom channel. Hence it is now possible to estimate the azimuth angle of the source, in addition to range and depth using a single vertical array. It should be noted that the effective slope of the bottom depends on the azimuth angle of the source with respect to the downslope (or upslope) direction. The effective slope is zero in the strike direction or perpendicular to the downslope direction.

Next, we consider a two slope sea bottom, namely, continental margin and deep sea (see Fig. 1). The source is on the deep sea side and the array in the continental margin. Since the shallow sea is on the opposite side we shall assume that insignificant signal enegy would be returned by the shallow end. The image structure shown in Fig. 6 is a result of reflection from both sloping sea bottom and flat deep sea bottom.

\section{Numerical Results :}

Azimuth estimation:

Figure(4) illustrates the effect of azimuth. It can be observed that the main peak becomes increasingly narrow as we approach $90 \mathrm{deg}$ azimuth angle, that is down slope and beyond this it becomes once again broader. Note that the peak height remains same in all experiments however the background pattern is different.

\section{Effect of wedge angle:}

It is possible to estimate the source azimuth in a sloping bottom channel but not in a flat bottom channel. Hence, it is natural to expect an improved performance as we increase the slope. Fig 5 shows the effect of bottom slope on the performance of MISA. Azimuth estimation for different slope angles are shown. It can be observed from the figure that the peak height remains constant as we increase the slope and that the algorithm performs better at higher slope angles. This can attributed to the fact that the aperture of the source distribution increases as we increase the slope angle. Figure(9) shows the image distribution for two different slopes. It can be observed that the same number of critical images occupy a bigger arc of the circle, for higher slope. 
Effect of finite data:

Fig 6 shows the effect of finite data on MISA. When source spectral matrix is exactly known(theoretical), the orthogonality condition is perfectly satisfied. Hence the signal. peak height becomes infinite. But in the finite data case. only an estimate of the spectral matrix is known. Hence, the orthogonality condition is only approximately satisfied. Thi::, peaks will not have a infinite value but a finite large value. Plot of peak height for different data length is shown in Fig 6 . it can be observed from the graph that the peak value increases at the rate of $2 \mathrm{~dB}$, for each doubling of the number of snapshots. It is interesting to observe that the background pattern remains almost constant, as we increase the number of snapshots. The height of the largest sidelobe was found to be constant in all the experiments as shown in Fig

\section{Conclusion:}

It is shown that when the channel bottom is sloping. the radial symmetry of the flat bottom channel is destroyed. Hence location of the images with respect to the receiver is a function of the range, depth and azimuth, except when the receiver is at apex. This enables us to measure the azimuth in addition to range and depth using single vertical array of sensors. Simulations showed that azimuth scan is very sensitive to the slope of the channel. Better performance is obtained for higher slope angles. Also, the azimuth scan was found to be most sensitive when the source is perpendicular to the slope direction. The finite data studies show that peak height increases as we increase the snapshots. the algorithm works reasonably well even for small number of snapshots, for example, ten snapshots.

\section{REFERENCES}

[1] C.S.Clay, "Optimum time domain signal transmission and source location in a waveguide," Journal of the Acoustical Society of America, Vol.81, No.3, pp.600-664 March 1987.

[2] S.li and C.S.Clay, "Optimum time domain signal transmission and source location in a waveguide: Experiments in a ideal wedge waveguide," Journal of the Acoustical Society of America, Vol.82, No.4, pp. 1409-1417 October 1987.

[3] P.G. Krishna Mohan, "Source location by signal subspace approach and ambient noise modelling in shallow water," IISC Ph.D. thesis, January 1989

[4] A.H. Yaglom, Introduction to theory of stationary random functions, Prentice-Hall, Englewood Cliffs, 1962.,Ch. 2, pp

\section{Figures}

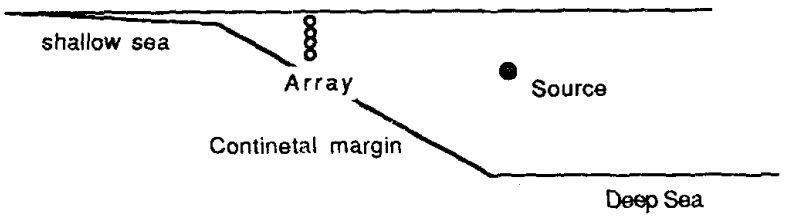

Fig. 1: A sound source in the continental margin and a vertical array of sensors.
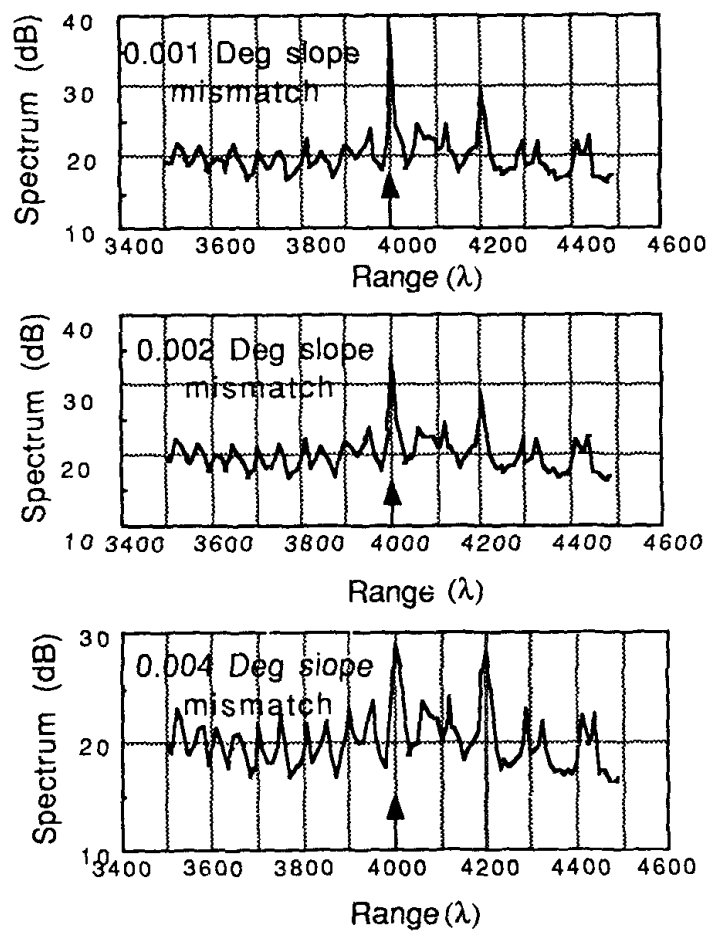

Fig. 2: The effect of a gentle bottom slope on the performance of MISA designed for horizontal bottom.

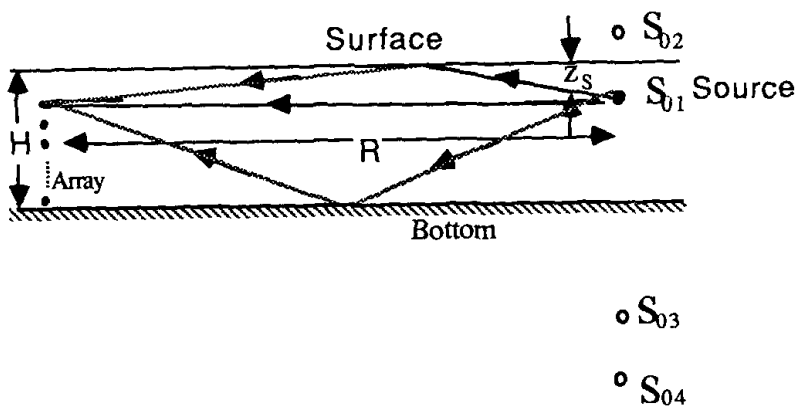

Fig. 3: A point source between two reflecting surfaces and its repeated images lying on a vertical line passing through the source. A Pekeris model is assumed for the shallow water channel. 


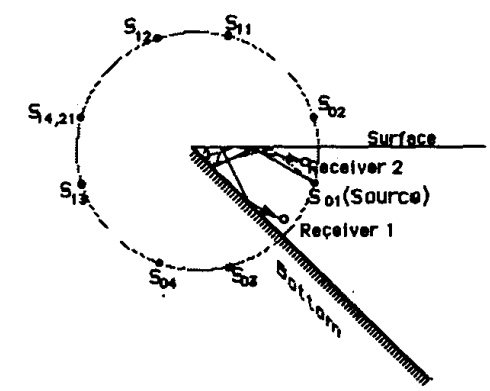

Fig 4: Image structure in a wedge shaped shallow channel. Ray paths for two receiver positions are channel. Ray paths for two receiver positions are
shown for the source in the downslope direction.

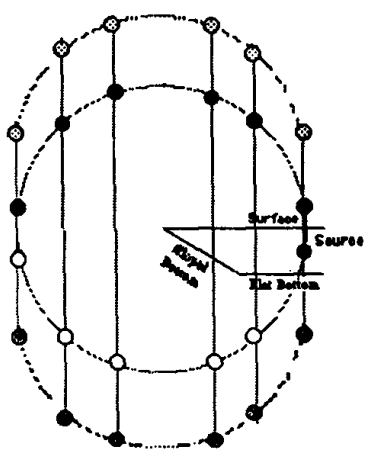

Fig 6: Image structure due to two reflection surfaces. The sloping surface distributes the images on a
circle. The flat surface repeatedly reflects the images produced by the sloping surface.

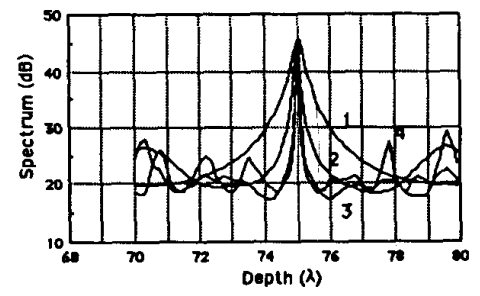

Fig 8: Effect of wedge angle.

Curve 1: $-0.5^{0}$, Curve 2: $-1.0^{0}$,

Curve 2: $-3.0^{0}$, Curve 4: $-5.0^{\circ}$

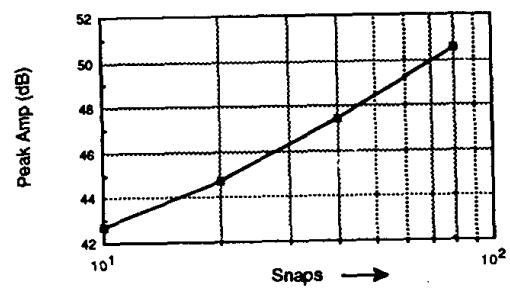

Fig.10. Difference between the peak to highest sidelobe in dBs is plotted against the number of snapshots.
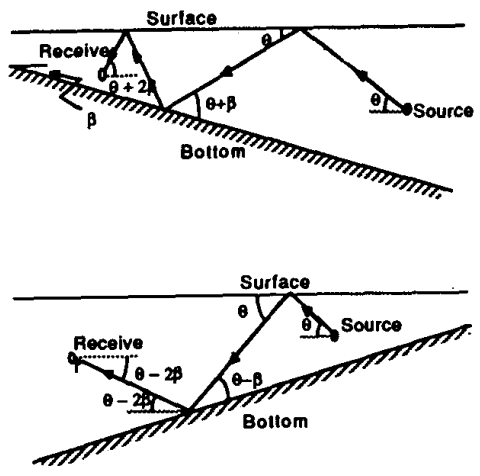

Fig. 0 A typical ray path in a wedge shaped shallow A typical ray path in a wedge shapod thallow
sea.(a)Source is in the downslope direction sea.(a)Source is in the downslope direction
(b)Sounce is in the upslope direction.
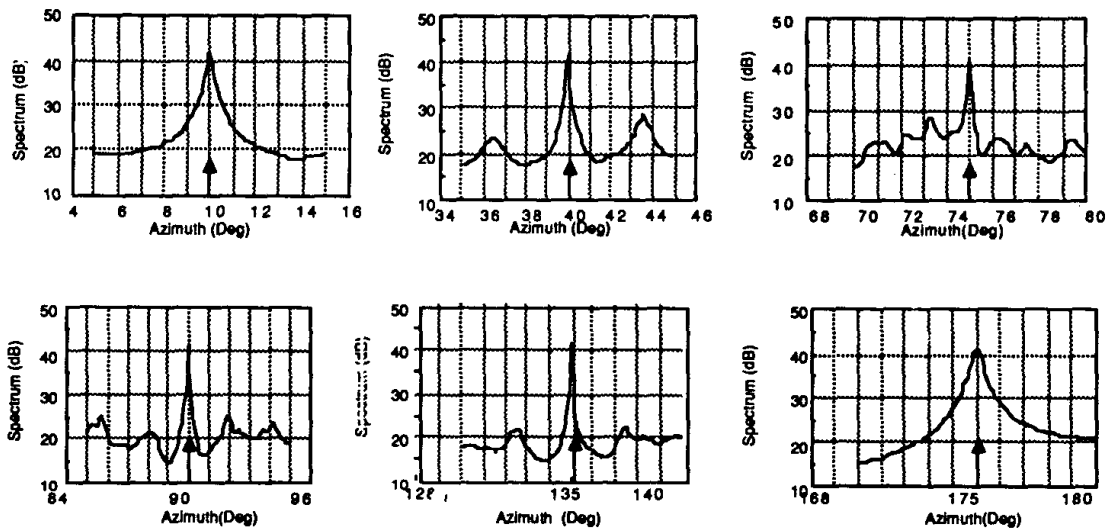

Fig. 7: Effect of source azimuth angle in azimuth estimation.

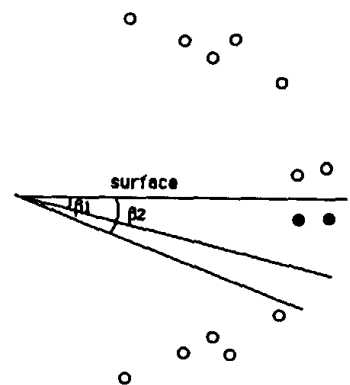

Fig. 9: image distribution for two different slopes. 